E3S Web of Conferences 1, 13008 (2013)

DOI: $10.1051 / \mathrm{e} 3$ sconf/20130113008

(c) Owned by the authors, published by EDP Sciences, 2013

\title{
Considering bioavailability in the remediation of heavy metal contaminated sites
}

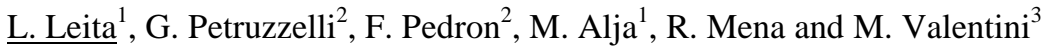 \\ ${ }^{1}$ CRA Agricultural Research Council Research, Centre for Plant Soil System, Via Trieste 2334170 Gorizia Italy \\ liviana.leita@entecra.it; alja.margon@entecra.ir \\ ${ }^{2}$ CNR Institute of Ecosystem Study Via Moruzzi 156124 Pisa Italy, petruzzelli@ise-cnr.it; francesca.pedron@ise-cnr.it \\ ${ }^{3}$ CRA Agricultural Research Council Research Centre for Plant Soil System, Instrumental Centre of Tor Mancina, S.P. \\ Pascolarese - Strada della Neve Km 1, 00015 Monterotondo, Rome, Italy massimiliano.valentini@entecra.it; \\ mena.ritota@entecra.it
}

\begin{abstract}
Many years of research have demonstrated that instead of the total concentration of metals in soil, bioavailability is the key to understand the environmental risk derived by metals, since adverse effects are related only to the biologically available forms of these elements. The knowledge of bioavailability can decrease the uncertainties in evaluating exposure in human and ecological risk assessment. At the same time, the efficiency of remediation treatments could be greatly influenced by availability of the contaminants. Consideration of the bioavailability processes at contaminated sites could be useful in site-specific risk assessment: the fraction of mobile metals, instead of total content should be provided as estimates of metal exposure. Moreover, knowledge of the chemical forms of heavy metals in soils is a critical component in the evaluation of applicability of different remediation technologies such as phytoremdiation or soil washing.
\end{abstract}

Keywords: Heavy metals, bioavailability, remediation, soil

\section{Introduction}

Remediation and management of heavy metal contaminated sites is often technically difficult and can be very expansive when large volumes of contaminated material have to be treated. To optimise the limited environmental management and remediation resources, there is a need to improve risk assessment by including more reliable standards, consistent with the specificity of the contaminated site. For this purpose, pointing out and evaluating "how clean is clean?" (e.g. site-specific concentration levels to which a contaminant has to be reduced) are noteworthy for achieving realistic risk assessments and remediation endpoints together with the more suitable remediation-strategy to be adopted. However, the ability to accurately determine the effects of contaminants on individual species, populations, communities, and ecosystems is hampered by uncertainty in the quantification of exposure of the individual species. This uncertainly has lead the regulatory authorities towards a precautionary and conservative approach to set out standards by assuming that the total amount of contaminants present in a given soil is available for uptake by the ecological receptors, thus leading to overestimate the risk. Explicitly incorporating bioavailability into the risk assessment process would offer the possibility to demonstrate that in some cases only a fraction of the contaminant's total mass actually has the potential to enter possible receptors. In other cases, better understanding of bioavailability processes can lead to more protective risk estimates, for example by identifying an important exposure pathway that has been previously overlooked. In Europe, the Governative Commission is still arranging a Directive on "soil thematic strategy for soil protection" and, among the documents drawn up by the involved task forces, the Soil Communication Paper by the Task Group on Contamination stated that (bio) availability must be included in the new European policy: 'Policies for soil protection should be flexible and take aspects of (bio)availability into account, especially for immobile persistent chemicals (metals, PAHs)'. At least consensus on the concept of bioavailability should be part of new policy on soil protection. The aim of this paper is to 
promote the consideration of bioavailability of heavy metals as a tool to select rationale remediation strategies at contaminated soils.

\section{Results and Discussion}

\section{Processes of bioavailability in soil}

As reported by Harmsen, organisms respond only to the fraction of contaminants that is biologically available (bioavailable) for organism. This is particularly true in soils that undergo interaction of contaminant molecules in such a way that the contaminant is not attainable by the organism or present in a non available form (sometimes referred to as sequestration or irreversible sorption). The bioavailable fractions of contaminants depend on soil properties and processes varying with time, due to ageing. Greater knowledge of the processes regulating bioavailability at a site specific level is therefore essential to understand the risks associated with pollution and can guide the decision-making processing terms of the choice of cleanup technologies.

Considering heavy metals, the passage from unavailable to available form is regulated by chemical, physical and biological conditions and time, which determine the transfer of metals from the solid to the solution phase that are governed by properties such as $\mathrm{pH}$, organic matter and clay content, cation exchange capacity redox potential etc. (Leita et al., 2009).

\section{The measurement of bioavailability}

One of the main difficulties in the application of the concept of bioavailability in remediation procedures lies in the lack of a clear consensus as to what methodology to use to measure bioavailability.

One possible strategy to overcome this obstacle is to apply a series of tests to assess bioavailability instead of looking for a universal method that is valid in all conditions. The only direct way of measuring the bioavailability of a contaminant for a certain organism would be to use that organism itself. The number of organisms in the natural ecosystem is obviously too large to manage this approach, and we therefore need to resort to approximations in the form of "test organisms" which supply, by means of biological tests, some indications for the real bioavailability.

On the other hand, if the limiting factor in the bioabsorption of a chemical compound derives from a series of chemical processes, indications as bioavailability can also come from chemical tests that identify the nature of the bonds that bind a certain substance to the surfaces of the soil, for example by using an extracting solution.

According to this concept, information on the bioavailability derives from laboratory tests in which bioavailability is determined both biologically and chemically. The chemical and biological tests alone do not define the bioavailability, both must be considered as tools, which provide information on bioavailability.

If we consider, for example, phytoextraction of heavy metals, the processes that determine bioavailability are: the release from the solid phase into the liquid phase, and the uptake of the element in soluble forms by the roots. Bioavailability tests therefore need to consider two distinct aspects: the physiochemical solubilization process and the physiological uptake process. Soil characteristics and plant characteristics determine bioavailability.

This multiple approach for the determination of of the bioavailability processes is an essential aspect of the ISO international standard 17402/2008 on bioavailability and it properly agrees with the indications of EPA, which suggests the use of the "weight of evidence" (WOE) criterion as a support tool for evaluating the contributions from different tests:

- Relevance in terms of soil chemistry.

- Relevance in terms of the final receptor.

- Relevance in terms of the environmental path

- Acceptability and validation of the method.

Chemical and biological tests provide different lines of evidence regarding the processes of bioavailability in a specific site and the concept of WOE is used to refer to how the results of various combined tests should be used.

\section{Bioavailability as a tool in remediation strategies}

Knowledge of bioavailability processes can be used at various stages in the selection and application of remediation technologies. Two different strategies, can be applied - either the reduction or increase of bioavailability.

Techniques that reduce bioavailability have the aim of preventing the movement of pollutants from the soil to the living organism essentially by:

a) Removal of the labile phase of the contaminant, that is of that fraction which is intrinsic to the processes of bioavailability.

b) Conversion of the labile fraction into a stable fraction (for example, the precipitation of metals), or modification of the redox state towards insoluble forms.

There are also procedures that aim to increase the bioavailability of pollutants, which can be used in the frame of technologies, which remove the solubilized contaminants.

Understanding of the bioavailability of the pollutants plays a primary role in the assessment of the applicability and efficiency of biological technologies. For example, in heavy metals phytoextraction the contaminants must be in a mobile form available to be absorbed by the plant root systems and chelating agents are used to solubilize metals (Doumett et al., 2011). On the other hand, if a process of phytostabilisation is needed, the pollutants must be, 
essentially, present in insoluble forms that are not bioavailable

The knowledge of chemical forms of the metals in the soil is of particular importance for "in situ" technologies such as "soil washing", to choose the type of liquid phase needed to carry out the washing process. In fact, in the case of heavy metals in prevalently insoluble forms, it is advisable to use water as a washing fluid due to its low cost, its easy recovery, its limited corrosive action on machinery and its overall ease of use .

In the case of soil flushing, the amounts of soluble forms determines the need of $\mathrm{pH}$ adjustments, which facilitate the complete solubilisation of the contaminants. Finally, there can be unforeseen effects that some remediation techniques have on bioavailability. The most evident case is that of the excavation and removal of soil. During the phases of excavation and movement of contaminated soil, the exposition levels of workers are much higher than when the soil is maintained in situ. The finest particles can enter the atmosphere where even the most volatile contaminants $(\mathrm{Hg})$ can be dispersed becoming bioavailable when inhaled.

\section{Conclusions}

Inserting the concept of bioavailability in remediation schemes starting from the stage of risk assessment is particularly appropriate for heavy metals. For these contaminants the assumption that the total concentration in soil is bioavailable, is not scientifically reliable. An accurate assessment of the fate of heavy metals in soil and their bioavailability can help to select the best remediation technologies saving a considerable amount of time and resources. The factors that greatly determine the need to take into account the processes of bioavailability are:

- When only some chemical forms of the contaminants are a source of risk for the site.

- When default assumptions regarding bioavailability are not suitable because of the site's specific characteristics.

- When there is a substantial difference in the remediation goals if the bioavailability of heavy metals are taken into account.

- When it is foreseen that the final destination of the site will not be modified at least in the near future.

In many cases, there is a precise understanding of the mechanisms and reactions governing heavy metal bioavailability, whilst for others there is still uncertainty and further studies and in-depth analyses are required. The scientific community is making a great effort to widen our understanding of bioavailability processes, in order to make them more widely used in risk the assessment procedures, following some fundamental guidelines:

- Select appropriate measurement tools for determining heavy metal bioavailability.

- Understand, assess and when possible, reduce the uncertainty concerning the parameters and models used for the particular processes of bioavailability.

- Develop a monitoring plan to assess the possible, even if unlikely, time variations in bioavailability.

There is little likelihood of a variation in bioavailability that can be caused only by unforeseen sudden geochemical variations, changes in the use of the site or the introduction of a new species into the area. However, a monitoring program is a part of many remediation strategies and it is certainly not a conceptually complex aspect. To promote bioavailability as a practical tool in soil remediation, there is a need for methods able to combine chemical, biological and toxicological measures. Legislative aspects and public acceptance regarding bioavailability are very important aspects that do not fall within the scientific context. However, it is important to emphasize that when innovative remediation strategies are used, it is essential to involve from the beginning all the interested stakeholders, because only by information it is possible to obtain a greater acceptability.

\section{References}

Doumett S, Azzarello E, Fibbi D, Mancuso S, Mugnai S, Petruzzelli G, Del Bubba M. Influence of the application renewal of glutamate and tartrate on $\mathrm{Cd}, \mathrm{Cu}, \mathrm{Pb}$ and $\mathrm{Zn}$ distribution between contaminated soil and Paulownia tomentosa in a pilot-scale assisted phytoremediation study. Int $\mathbf{J}$ Phytoremediat 2011;13:1-17.

Harmsen J. Measuring bioavailability: from a scientific approach to standard methods. J of Environ Qual 2007;36:1420-1428.

ISO 17402 Soil quality-requirements and guidance for the selection of methods for the assessment of bioavailability of contaminants and soil materials, 2008.

Leita L, Margon A, Pastrello A, Arcon I, Contin M, Mosetti D. Soil humic acids may favour the persistence of hexavalent chromium in soil. Environ Pollut 2009;157:1862-1866. 